\title{
Control of Differential Air and Hydrogen Pressures in Fuel Cell Systems*
}

\author{
Yoshitomo ASAI** and Nobutaka TAKAHASHI*** \\ ** EV System Laboratory, Nissan Research Center, NISSAN MOTOR CO., LTD. \\ 1, Natsushima-cho, Yokosuka-shi, Kanagawa 237-8523, Japan \\ E-mail: yoshitomo-asai@mail.nissan.co.jp \\ *** Integrated System Group, Electronics Quality Engineering Department, Electronics Engineering \\ Development Division, NISSAN MOTOR CO.,LTD \\ 560-2, Okatsukoku, Atsugi-shi, Kanagawa 243-0192, Japan
}

\begin{abstract}
This paper describes a method of configuring a control system for keeping the differential pressure of the hydrogen and air supplied to a fuel cell within a specified range. The proposed method assumes that the control system of the air pressure and mass flow rate is based on the use of a mathematical model, whereas the hydrogen pressure control system does not employ a mathematical model and treats the consumption of hydrogen during power generation as a disturbance. When hydrogen consumption due to power generation is treated as a disturbance, the responsiveness of the hydrogen pressure and air pressure varies depending on the amount of power being generated. Therefore, the air pressure is predicted using transfer functions and the hydrogen pressure is made to follow the predicted value. In addition, the air pressure is made to follow the larger of either the reference pressure or the hydrogen pressure. This paper first describes the derivation of the mathematical model of the air supply system. The mathematical model is then used to design a sliding mode control system based on the two variables of the air pressure and mass flow rate. The method of configuring the differential air and hydrogen pressure control system is then explained, and experimental results are presented to validate the proposed design method.
\end{abstract}

Key words : Fuel Cell, Automobile, Modeling, Transient Response

\section{Introduction}

Global warming has been proceeding in recent years due to rising levels of greenhouse gases (GHGs) in the atmosphere such as carbon dioxide (CO2). This issue has attracted increasing attention along with concern about peak oil. The IPCC Fourth Assessment Report (1) states that the concentration of GHGs in the atmosphere must be kept below at least 535 ppm in order to hold the increase in the global temperature since the Industrial Revolution to less than $2.8^{\circ} \mathrm{C}$.

In view of this situation, Nissan unveiled a medium-term environmental action plan in 2006 called the Nissan Green Program 2010. One of the goals of this plan is to step up the development of technologies for electrified vehicles and to promote their introduction and widespread use. Toward that end, efforts are under way to develop fuel cell vehicles (FCVs) and electric vehicles (EVs) that provide improved performance.

If an FCV is to generate power stably and promptly in response to the driver's demands, the pressure and mass flow rate of the air and the pressure of the hydrogen supplied to the fuel cell must follow given reference values accurately and quickly. Another requirement is that differential air and hydrogen pressures must be kept within a specified range in order to avoid any decline in fuel cell durability and performance. To meet these requirements, a feedback control system is employed for the air pressure and mass flow rate and also for the hydrogen

${ }^{*}$ Received 28 Apr., 2010 (No. 10-0185)

[DOI: 10.1299/jsdd.5.109]

Copyright $\subset 2011$ by JSME 
pressure so as to avoid any deviation from the reference values under steady-state operation. However, when these feedback control systems are designed independently, it is difficult to keep the differential air and hydrogen pressures within the specified range.

Since recent FCVs are equipped with a high-pressure hydrogen tank for supplying hydrogen to the fuel cell, the hydrogen pressure can be raised more quickly than the air pressure. On the other hand, the consumption of hydrogen in the generation of power is the dominant factor in the operation of reducing the pressure. Accordingly, the pressure reduction response varies depending on the state of power generation. The air supply system uses a compressor to compress air taken in from the atmosphere, so it takes more time to raise the air pressure than the hydrogen pressure. On the other hand, as will be described later, the air pressure can be reduced quickly by opening the pressure valve installed downstream of the fuel cell and evacuating air. The fact that the response of the hydrogen pressure and air pressure differs according to the power generation state and whether the pressure is increasing or decreasing complicates the difficulty of maintaining differential pressures within the specified range.

Fuel cell control systems are being studied by researchers all over the world. Pukrushpan et al. ${ }^{(3)}$ and Lin et al. ${ }^{(4)}$ constructed precise models of a fuel cell system and applied optimal control theory to investigate the performance obtained for controlling the air pressure and mass flow rate. Reuter et al. created a nonlinear model to express the compressor dynamics and validated the model and control performance by using PID control. ${ }^{(5)}$ Danzer at al. ${ }^{(6)}$ and Wang et al. ${ }^{(7)}$ treated load changes as disturbances for the control system and proposed control systems based on H-infinity control that are capable of supplying the air flow needed for generating power even if the power generation load fluctuates.

However, all of these studies were conducted by computer simulation. The literature does not show any examples of experimental validation of control performance for a fuel cell system that can be fitted to an actual vehicle. To the best of our knowledge, there are no examples of studies in which attempts were made to control differential hydrogen and air pressures and to validate the effectiveness of the control system experimentally. Against this backdrop, this paper describes a design method for a differential pressure control system based on the controllers used to regulate the air pressure and mass flow rate and hydrogen pressure in a fuel cell system. It also presents the results of experiments conducted to validate the effectiveness of the proposed design method.

The authors have previously proposed a control method of the air pressure and flow in a fuel cell system using two-variable and two-degree-of-freedom sliding model control ${ }^{(2)}$. While the air pressure and flow can be controlled using this proposed method, the differential air and hydrogen pressures in a fuel cell system must be controlled within a specified range to maintain the durability of the fuel cell membrane. This paper describes a method of configuring a differential air and hydrogen pressure control system based on the previously proposed technique and presents experimental results that validate the proposed design method.

\section{System configuration}

Figure 1 is a schematic diagram showing the configuration of a proton exchange membrane fuel cell (PEM-FC). The separator, which seals the cell, guides the fuel stream to the electrodes and connects each cell electrically, is made of carbon. The electrodes are also made of carbon with platinum as the catalysts. This fuel cell system can generate current in a range from $0 \mathrm{~A}$ to a maximum of about $300 \mathrm{~A}$. It produces maximum power of $90 \mathrm{~kW}$.

The configuration of the air supply system is shown in Fig. 2 along with factors that affect the air pressure and mass flow rate. Intake air is compressed by a compressor and cooled by an air cooler through heat exchange with the coolant. Product water formed during power generation is transferred via a dehumidifier to a humidifier and added to the air as water vapor. Oxygen consumed in the power generation process at the cathode according to Eq. (1) is extracted from the air supply system. The air supply system also produces water that forms in the power generation process, and heat exchange takes place between the fuel cell and the 
air according to the reaction in Eq. (1). The air supply system also incorporates a ventilation line and an air bypass valve that are needed to make the fuel cell system viable. The operation of the latter valve is unrelated to the control system for the air pressure and mass flow rate.

\section{Cathode}

$$
2 \mathrm{H}^{+}+\frac{1}{2} \mathrm{O}_{2}+2 e^{-} \rightarrow \mathrm{H}_{2} \mathrm{O}
$$

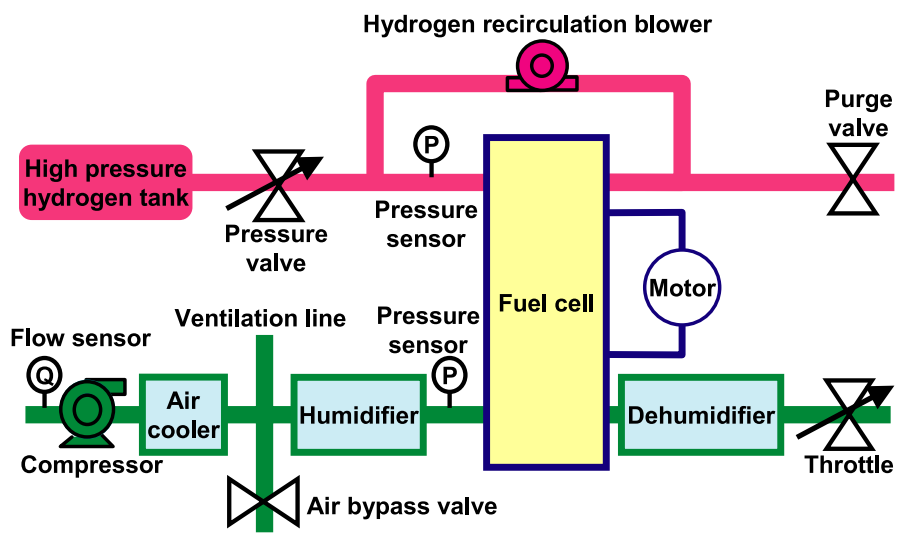

Fig. 1 Configuration of fuel cell system

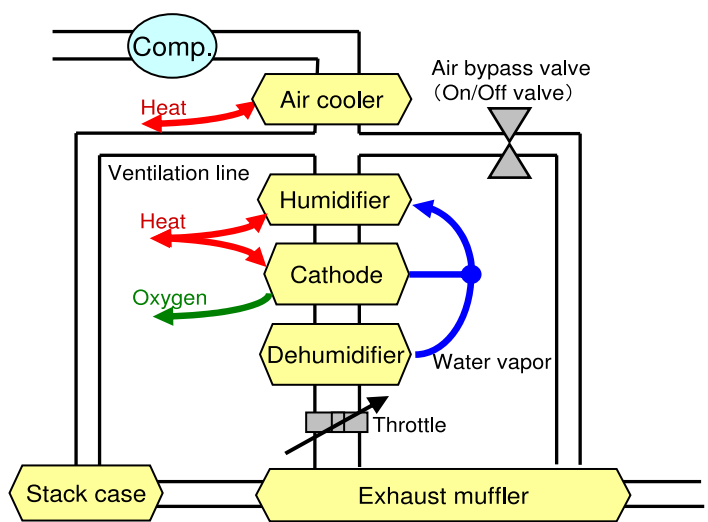

Fig. 2 Configuration of air supply system

The hydrogen supply system includes a high-pressure hydrogen storage tank, a pressure valve that regulates the flow of hydrogen supplied from the tank, a hydrogen recirculation blower for returning and reusing hydrogen that is not consumed during power generation, and a purge valve for exhausting nitrogen that migrates via the electrolyte membrane from the cathode to the anode. Hydrogen is consumed in the power generation process at the anode according to Eq. (2) and is extracted from the hydrogen supply system.

Anode

$$
H_{2} \rightarrow 2 H^{+}+2 e^{-}
$$

The actuators used in the air supply system are the compressor and the air pressure valve (throttle), while those used in the hydrogen supply system are the hydrogen pressure valve, hydrogen recirculation blower and nitrogen purge valve. The operation of the hydrogen recirculation blower and the nitrogen purge valve is unrelated to the control inputs and controlled variables of the hydrogen pressure control system. For that reason, only the hydrogen pressure valve is treated as an actuator for controlling the hydrogen pressure. Since hydrogen and air are consumed in the power generation process of a fuel cell, as indicated in Eqs. (1) and (2), the amount of power generated also influences the hydrogen pressure and air pressure. The 
controlled variables are the air pressure at the cathode inlet, the air mass flow rate into the compressor and the hydrogen pressure at the anode inlet. They are also observed variables that are monitored with sensors.

Figure 3 is a block diagram of the plant from the controller to the air supply system that is the object of control. The notations $z^{-n}$ and $z^{-m}$ in the figure denote the respective delay in the communication lines. The compressor control module consists of a motor and an inverter, while the throttle control module comprises a control system for the throttle. The air supply system indicates the air supply system shown in Fig. 2.

The notation $T_{c}$ in Fig. 3 is a table that converts the air mass flow rate signal into a rotational speed as the command for the compressor control module. The notation $T_{v}$ is also a table that converts a signal, expressing the opening ratio of the cross-sectional area of the pipeline, to a throttle angle command signal for input into the throttle control module. In this paper, $T_{c}, T_{v}, z^{-n}, z^{-m}$, the compressor control module and the air supply system are treated as the plant, and the outputs from the control system for the air pressure and mass flow rate are the signals input into $T_{c}$ and $T_{v}$.

Pressure in the hydrogen supply system is controlled by PID control without creating a mathematical model.

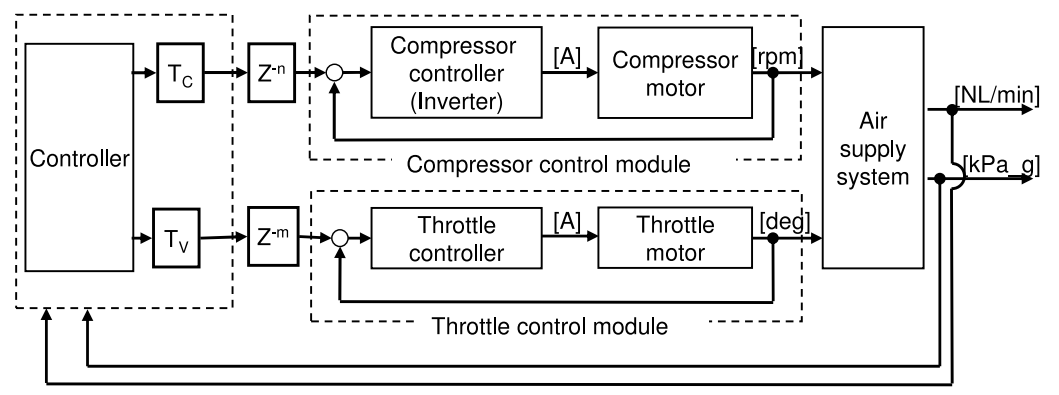

Fig. 3 Block diagram of plant

\section{Design of air pressure and mass flow rate control system}

\subsection{Modeling of air supply system}

The air supply system was modeled as shown in Fig. 4, assuming the system configuration in Fig. 2. First, the volume from the compressor outlet to the cathode inlet was assumed to be a single volume element on the supply side (supply manifold). The volume from the cathode outlet to the throttle was then expressed as the exhaust side volume element (return manifold). The pressure loss in each part was represented as an orifice diameter, and the heat exchange between the interior and exterior of the system was assumed to be constant in each part.

In deriving the linear model, $T_{c}$ and $T_{v}$ in Fig. 3 were treated as linear, steady-state gains, and $z^{-n}, z^{-m}$ and the dynamic characteristics of the throttle control module were ignored as being sufficiently faster than the pressure dynamics. The effect of oxygen consumption during power generation on the air pressure was also ignored because it is compensated for by the robustness of the feedback control system. The mass flow rates through the air bypass valve and ventilation line were ignored as being small compared with the mass flow rate in the pipeline to the cathode. Considering the operating temperature of the fuel cell, the influence of saturated water vapor on pressure fluctuations can be ignored as being negligibly small. Because little pressure loss occurs in the exhaust muffler, the muffler pressure was assumed to be atmospheric. 


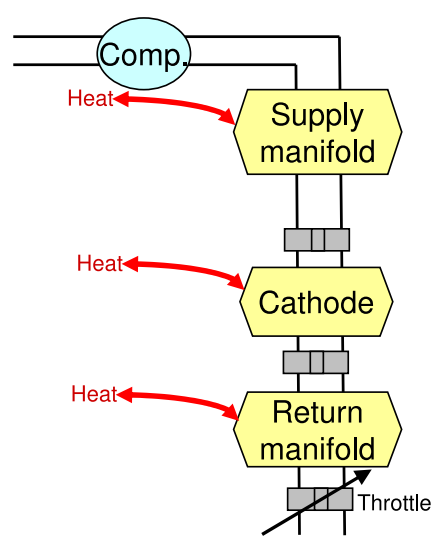

Fig. 4 Configuration of plant model

\subsection{Flow characteristics around orifice}

The mass flow rate passing through an orifice is calculated from Eqs.(3) and (4). The pressure ratio expressed in Eq. (5) divides the flow characteristic. When Eq. (5) is satisfied, Eq. (4) is used for the calculation. Here, $C$ is the discharge coefficient of the orifice, $A_{T}$ is the opening area of the orifice, $p_{1}$ is the upstream absolute pressure, $p_{2}$ is the downstream absolute pressure, $T_{1}$ is the upstream absolute temperature, and $R_{a}$ is a gas constant. $\gamma$ is the specific heat ratio of air.

$$
\begin{aligned}
& W=\frac{C A_{T} p_{1}}{\sqrt{R_{a} T_{1}}}\left(\frac{p_{2}}{p_{1}}\right)^{\frac{1}{\gamma}}\left\{\frac{2 \gamma}{\gamma-1}\left[1-\left(\frac{p_{2}}{p_{1}}\right)^{\frac{\gamma-1}{\gamma}}\right]\right\}^{\frac{1}{2}} \\
& W_{\text {choked }}=\frac{C A_{T} p_{1}}{\sqrt{R_{a} T_{1}}} \gamma^{\frac{1}{2}}\left(\frac{2}{\gamma+1}\right)^{\frac{\gamma+1}{2(\gamma-1)}} \\
& \frac{p_{2}}{p_{1}} \leq\left(\frac{2}{\gamma+1}\right)^{\frac{\gamma}{\gamma-1}}
\end{aligned}
$$

\subsection{Linearization of mass flow rate around orifice ${ }^{(3)}$}

Although Eqs. (3) and (4) have strong non-linearity, the pressure difference between the upstream volume and the downstream volume is small, so the mass flow rate can be calculated with a linearized form of Eq. (6). Here, $g$ is the nozzle constant. For example, Fig. 5 shows plots calculated with Eqs. (3), (4) and (6) for various upstream manifold pressures. If Eq. (5) is satisfied, Eq. (4) is used for the calculation. Since Fig. 5 shows the general characteristics of the flow around an orifice, the unit of the Y-axis of the figure is not related to the specifications of the fuel cell system in Fig. 1. Since the dynamics of mass flow is faster than the pressure dynamics of the volumes, the former dynamics can be ignored.

$$
W \simeq g\left(p_{1}-p_{2}\right)
$$

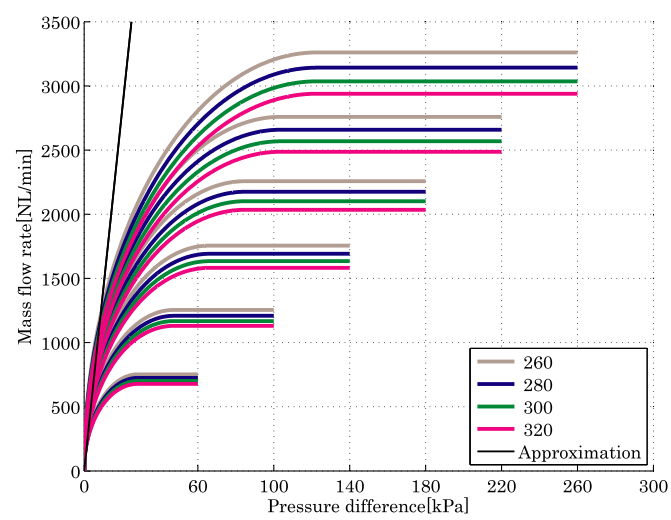

Fig. 5 Linearization of flow 


\subsection{Pressure dynamics}

The pressure dynamics is derived from the energy conservation law, the ideal gas law, and the air thermodynamic properties as Eq. (7). Here, $W_{1}$ is the mass flow rate out of the upstream manifold, $W_{2}$ is the mass flow rate into the downstream manifold, $T_{1}$ is the absolute temperature of the upstream volume, $T_{2}$ is the absolute temperature of the downstream volume and $n$ is a polytropic index. When $n$ is equal to 1 , the equation becomes an isothermic relation. If $\mathrm{n}$ is the specific heat of ratio of air $\gamma$, Eq. (7) becomes an adiabatic relation ${ }^{(3)}$.

$$
\frac{d p}{d t}=\frac{n R_{a}}{V}\left(W_{1} T_{1}-W_{2} T_{2}\right)
$$

\subsection{Derivation of linear model of air supply system}

The compressor dynamics was treated as a second-order system that approximated the dynamics of the flow model from the input to $T_{c}$ to the output flow of the air supply system in Fig. 3. The parameters of the model were identified on the basis of simulated and experimental results. The equation for flow through an orifice was linearized around the operating point. A linear model of the air supply system was then derived as shown in Eq. (8) using the variables in Table 1.

$\left\{\begin{aligned} \frac{d}{d t} \boldsymbol{x}_{0} & =\boldsymbol{A}_{0} \boldsymbol{x}_{0}+\boldsymbol{B}_{1} \boldsymbol{X}_{p} \boldsymbol{u}_{0} \\ \boldsymbol{y}_{0} & =\boldsymbol{C}_{0} \boldsymbol{x}_{0}\end{aligned}\right.$

$\boldsymbol{A}_{0}=\left[\begin{array}{ccccc}0 & 1 & 0 & 0 & 0 \\ a_{21} & a_{22} & 0 & 0 & 0 \\ a_{31} & 0 & a_{33} & a_{34} & 0 \\ 0 & 0 & a_{43} & a_{44} & a_{45} \\ 0 & 0 & 0 & a_{54} & a_{55}\end{array}\right]$

$\boldsymbol{B}_{1}=\left[\begin{array}{cc}0 & 0 \\ 1 & 0 \\ 0 & 0 \\ 0 & 0 \\ 0 & b_{52}\end{array}\right]$

$a_{21}=-\omega_{n}^{2}$

$a_{22}=-2 \zeta \omega_{n}$

$a_{31}=\frac{n R_{a}}{V_{s m}} T_{c p} \omega_{n}^{2}$

$a_{33}=-\frac{n R_{a}}{V_{s m}} T_{s m} g_{s m}$

$a_{34}=\frac{n R_{a}}{V_{s m}} T_{s m} g_{s m}$

$a_{43}=\frac{n^{\prime} R_{a}}{V_{c a}} T_{s m} g_{s m}$

$a_{44}=-\frac{n^{\prime} R_{a}}{V_{c a}}\left(T_{c a} g_{c a}+T_{r m} g_{r m}\right)$

$a_{45}=\frac{n^{\prime} R_{a}}{V_{c a}} T_{c a} g_{c a}$

$a_{54}=\frac{n^{\prime \prime} R_{a}}{V_{r m}} T_{c a} g_{c a}$ 


$$
\begin{aligned}
& a_{55}=-\frac{n^{\prime \prime} R_{a}}{V_{r m}} T_{c a} g_{c a} \\
& b_{52}=-\frac{n^{\prime \prime} R_{a}}{V_{r m}} T_{r m} g_{r m} \\
& \boldsymbol{x}_{0}=\left[\begin{array}{lllll}
x_{1} & x_{2} & p_{s m} & p_{c a} & p_{r m}
\end{array}\right]^{T} \\
& \boldsymbol{X}_{p}=\left[\begin{array}{cc}
1 & 0 \\
0 & p_{r m}
\end{array}\right] \\
& \boldsymbol{u}_{0}=\left[\begin{array}{l}
u_{c p} \\
k_{r m}
\end{array}\right] \\
& \boldsymbol{C}_{0}=\left[\begin{array}{ccccc}
\omega_{n}^{2} & 0 & 0 & 0 & 0 \\
0 & 0 & 0 & 1 & 0
\end{array}\right]
\end{aligned}
$$

Where $x_{1}$ and $x_{2}$ are the state variables of the flow model derived as a second-order system and $p_{s m}, p_{c a}$ and $p_{r m}$ are gage pressures.

The influence of the pressure change at the compressor outlet is compensated for by the robustness of the feedback controller, as the change can be assumed to be zero in the model. Moreover, in deriving the linear model, $p_{r m}$ of the bilinear element $\boldsymbol{X}_{p}$ was assumed to be a time-invariant $\boldsymbol{B}$ matrix having a constant value.

Table 1 Modeling constants and variables

\begin{tabular}{|c|c|}
\hline Symbol & Meaning \\
\hline$p_{s m}$ & supply manifold pressure \\
\hline$p_{c a}$ & cathode manifold pressure \\
\hline$p_{r m}$ & return manifold pressure \\
\hline$V_{s m}$ & supply manifold volume \\
\hline$V_{c a}$ & cathode volume \\
\hline$V_{r m}$ & return manifold volume \\
\hline$g_{s m}$ & cathode outlet orifice constant \\
\hline$g_{c a}$ & return manifold outlet orifice constant \\
\hline$g_{r m}$ & air temperature of expelled from compressor \\
\hline$T_{c p}$ & cathode volume air temperature \\
\hline$T_{s m}$ & return manifold air temperature \\
\hline$T_{c a}$ & polytropic indexes \\
\hline$T_{r m}$ & specific heat ratio of air \\
\hline$n, n^{\prime}, n^{\prime \prime}$ & damping ratio of compressor model \\
\hline$\gamma$ & control input to flow model \\
\hline$\zeta$ & open area ratio of throttle valve \\
\hline$\omega_{n}$ & natural angular frequency of compressor model \\
\hline$u_{c p}$ & cold air temperature \\
\hline$k_{r m}$ & \\
\hline
\end{tabular}

\subsection{Design of continuous sliding mode control system}

This section describes the design of the control system by applying continuous sliding mode control theory to extend the system in Eq. (12), which was constructed by adding one servo system each for pressure and the mass flow rate to the linear model derived in the previous section.

$$
\frac{d}{d t} \boldsymbol{x}=\boldsymbol{A x}+\boldsymbol{B u}+\boldsymbol{E r}
$$




$$
\boldsymbol{y}=\boldsymbol{C x}
$$

The sliding mode control system was designed by applying the final sliding mode method and the hyperplane design method using the zero point of the system. The control laws are given in Eqs. (13)-(17). The details of the design method can be found in references (8) and (9).

$$
\begin{aligned}
& \begin{array}{l}
\boldsymbol{u}=\boldsymbol{u}_{n l}+\boldsymbol{u}_{e q} \\
\boldsymbol{u}_{n l}=-(\boldsymbol{S B})^{-1} \boldsymbol{K} \operatorname{sat}\left[\boldsymbol{\phi}^{-1} \boldsymbol{\sigma}\right] \\
\boldsymbol{u}_{e q}=-(\boldsymbol{S B})^{-1}(\boldsymbol{S} \boldsymbol{A} \boldsymbol{x}+\boldsymbol{S E \boldsymbol { r }}) \\
\boldsymbol{\sigma}=\boldsymbol{S} \boldsymbol{x}=\boldsymbol{R}^{-1} \boldsymbol{B}^{T} \boldsymbol{P} \boldsymbol{x} \\
\text { sat }\left[\boldsymbol{\phi}^{-1} \boldsymbol{\sigma}\right]=\left\{\begin{array}{ccc}
\phi_{1}^{-1} \sigma_{1} & \text { for } & \left\|\sigma_{1} \leq \phi_{1}\right\| \\
\operatorname{sgn}\left(\phi_{1}^{-1} \sigma_{1}\right) & \text { for } & \left\|\sigma_{1}>\phi_{1}\right\| \\
\phi_{2}^{-1} \sigma_{2} & \text { for } & \left\|\sigma_{2} \leq \phi_{2}\right\| \\
\operatorname{sgn}\left(\phi_{2}^{-1} \sigma_{2}\right) & \text { for } & \left\|\sigma_{2}>\phi_{2}\right\|
\end{array}\right. \\
\boldsymbol{J}=\frac{1}{2} \int_{0}^{\infty}\left\{\boldsymbol{x}^{T}(t) \boldsymbol{Q x}(t)+\boldsymbol{u}^{T}(t) \boldsymbol{R} \boldsymbol{u}(t)\right\} d t
\end{array}
\end{aligned}
$$

where $\boldsymbol{S} \boldsymbol{x}=\mathbf{0}$ is a hyperplane, $\boldsymbol{R}$ is the weighting function of Eq. (18), $\boldsymbol{P}$ is the solution of the Riccati equation in Eq. (19), $\boldsymbol{K}$ is a switching gain matrix, with $\boldsymbol{K}=\operatorname{diag}\left[\begin{array}{ll}k_{11} & k_{22}\end{array}\right]$, and $\boldsymbol{\sigma}$ is a switching function.

$$
\boldsymbol{P A}+\boldsymbol{A}^{T} \boldsymbol{P}-\boldsymbol{P B} \boldsymbol{R}^{-1} \boldsymbol{B}^{T} \boldsymbol{P}+\boldsymbol{Q}=\mathbf{0}
$$

The saturation function in Eq. (17) for calculating the nonlinear throttle input varies according to the reference pressure $R_{p}$. Specifically, the width of the boundary layer is widened under a high pressure condition and narrowed under a low pressure condition, as illustrated in Fig. 6. This is done to counter the tendency for chattering to occur under a high pressure condition when the plant gain increases in relation to the mathematical model used in designing the control system. That happens because the linear model is derived assuming a fixed value of the bilinear component $\boldsymbol{X}_{p}$ in Eq. (8).

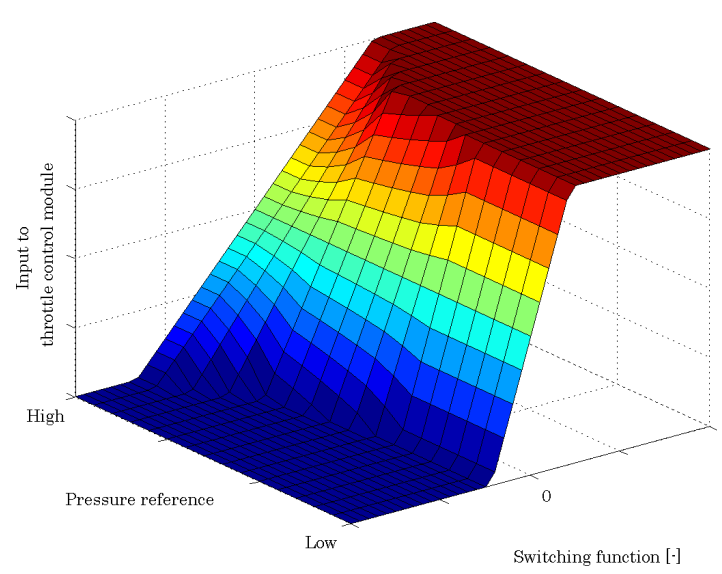

Fig. 6 Nonlinear input to the throttle control module

The air pressure at the cathode and the mass flow rate through the compressor are the only two state variables that can be observed in the plant. For the other state variables, optimal control theory was applied to design a minimal order linear observer using the duality of the system. 


\section{Preparation for configuring hydrogen/air pressure control system}

4.1. Transfer function from reference value to plant output in a 2 DOF control system using a minimal order observer

This section describes the derivation of the transfer function $\boldsymbol{G}$ from the reference value $\boldsymbol{r}_{0}$ to the air pressure $\boldsymbol{y}_{p}$ in a two-degree-of-freedom (2 DOF) control system using the minimal order observer shown in Fig. 7 and Eq. (20) below.

$$
\boldsymbol{y}_{p}=\boldsymbol{G} \boldsymbol{r}_{0}
$$

When the plant is defined by Eq. (21), the minimal order observer by Eq. (22) and the control input $\boldsymbol{u}$ is expressed as shown in Eq. (23), the error vector $\boldsymbol{e}$ by Eq. (24).

$$
\begin{aligned}
& \left\{\begin{array}{l}
\dot{x}_{0}=\boldsymbol{A}_{0} \boldsymbol{x}_{0}+\boldsymbol{B}_{0} \boldsymbol{u} \\
\boldsymbol{y}_{0}=\boldsymbol{C}_{0} \boldsymbol{x}_{0}
\end{array}\right. \\
& \left\{\begin{array}{l}
\dot{\boldsymbol{x}}_{p}=\hat{\boldsymbol{A}} \boldsymbol{x}_{p}+\hat{\boldsymbol{B}} \boldsymbol{y}_{0}+\hat{\boldsymbol{J}} \boldsymbol{u} \\
\hat{\boldsymbol{x}}_{0}=\hat{\boldsymbol{C}} \boldsymbol{x}_{p}+\hat{\boldsymbol{D}} \boldsymbol{y}_{0}
\end{array}\right. \\
& \boldsymbol{u}=-\boldsymbol{F}_{1} \boldsymbol{e}-\boldsymbol{F}_{2} \hat{\boldsymbol{x}}_{0}+\boldsymbol{V} \boldsymbol{r}_{0} \\
& \boldsymbol{e}=\int_{0}^{t}\left(\boldsymbol{r}_{0}-\boldsymbol{y}\right) d t
\end{aligned}
$$

By arranging Eqs. (21) and (22) and applying Eq. (26), we obtain Eq. (25), and Eq. (20) becomes Eq. (27).

$$
\begin{aligned}
& \left\{\begin{array}{l}
\frac{d}{d t} \grave{\boldsymbol{X}}=\tilde{\boldsymbol{A}} \grave{\boldsymbol{X}}+\tilde{\boldsymbol{E}} \boldsymbol{r}_{0} \\
\boldsymbol{y}_{p}=\tilde{\boldsymbol{C}} \grave{\boldsymbol{X}}
\end{array}\right. \\
& \tilde{\boldsymbol{A}}=\left[\begin{array}{ccc}
0 & -\boldsymbol{C}_{0} & 0 \\
-\boldsymbol{B}_{0} \boldsymbol{F}_{1} & \boldsymbol{A}_{0}-\boldsymbol{B}_{0} \boldsymbol{F}_{2} \hat{\boldsymbol{D}} \boldsymbol{C}_{0} & -\boldsymbol{B}_{0} \boldsymbol{F}_{2} \hat{\boldsymbol{C}} \\
-\hat{\boldsymbol{J}} \boldsymbol{F}_{1} & \hat{\boldsymbol{B}} \boldsymbol{C}_{0}-\hat{\boldsymbol{J}} \boldsymbol{F}_{2} \hat{\boldsymbol{D}} \boldsymbol{C}_{0} & \hat{\boldsymbol{A}}-\hat{\boldsymbol{J}} \boldsymbol{F}_{2} \hat{\boldsymbol{C}}
\end{array}\right] \\
& \tilde{\boldsymbol{E}}=\left[\begin{array}{c}
\boldsymbol{I} \\
\boldsymbol{B}_{0} \boldsymbol{V} \\
\hat{\boldsymbol{J}} \boldsymbol{V}
\end{array}\right], \tilde{\boldsymbol{C}}=\left[\begin{array}{lll}
0 & \boldsymbol{C}_{0}(2,:) & 0
\end{array}\right], \grave{\boldsymbol{X}}=\left[\begin{array}{c}
\boldsymbol{e} \\
\boldsymbol{x}_{0} \\
\boldsymbol{x}_{p}
\end{array}\right], \boldsymbol{r}_{0}=\left[\begin{array}{c}
r_{a f} \\
r_{a p 0}
\end{array}\right] \\
& \boldsymbol{y}_{p}=\boldsymbol{G} \boldsymbol{r}_{0}=\tilde{\boldsymbol{C}}(s \boldsymbol{I}-\tilde{\boldsymbol{A}})^{-1} \tilde{\boldsymbol{E}} \boldsymbol{r}_{0}
\end{aligned}
$$

where $C_{0}(2,:)$ is the second line of $C_{0}$.

\subsection{Application to a sliding mode servo control system with a minimal order observer}

A sliding mode control system is originally nonlinear. However, it can become a linear control system as shown in Eq. (28) within the boundary layer, when a saturation function is used. Assuming that the state variables are restricted in the boundary layer, $\boldsymbol{F}_{1}, \boldsymbol{F}_{2}$ and $\boldsymbol{V}$ in Eq. (29) can be found with Eqs. (26a) and (26b).

$$
\begin{aligned}
& \boldsymbol{u}=-(\boldsymbol{S B})^{-1}\left(\boldsymbol{S A} \tilde{\boldsymbol{X}}+\boldsymbol{S} \boldsymbol{E} \boldsymbol{r}_{0}\right)-(\boldsymbol{S B})^{-1} \boldsymbol{K} \boldsymbol{\phi}^{-1} \boldsymbol{S} \tilde{\boldsymbol{X}} \\
& {\left[\begin{array}{ll}
\boldsymbol{F}_{1} & \boldsymbol{F}_{2}
\end{array}\right]=(\boldsymbol{S B})^{-1}\left(\boldsymbol{S} \boldsymbol{A}+\boldsymbol{K} \boldsymbol{\phi}^{-1} \boldsymbol{S}\right)} \\
& \boldsymbol{V}=-(\boldsymbol{S B})^{-1} \boldsymbol{S E}
\end{aligned}
$$




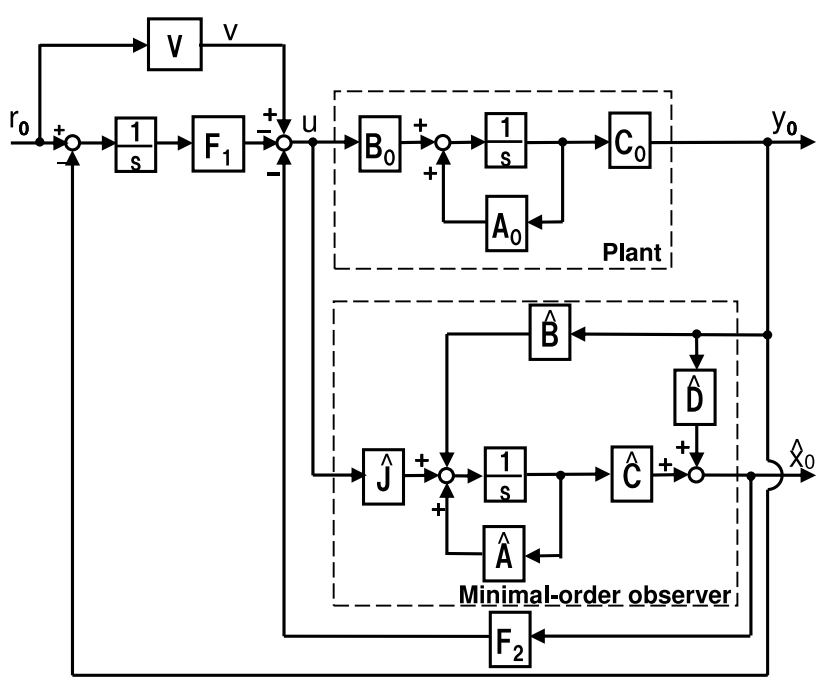

Fig. 7 Two-degree-of-freedom control using minimal-order observer

\section{Configuration of differential pressure control system}

The following assumptions were made for the configuration of the differential pressure control system.

( 1 ) Hydrogen pressure should display higher response under a condition of increasing pressure.

(2) It is not certain which system should display higher response under a condition of decreasing pressure.

The second assumption was made because the responsiveness of the hydrogen pressure under a decreasing pressure condition varies depending on the amount of power being generated. When a lot of power is being generated, much hydrogen is consumed in line with Eq. (2), and the hydrogen pressure exhibits higher responsiveness than the air pressure. In contrast, when little power is being generated, the responsiveness of the air pressure is higher because it can be reduced by simply opening the throttle. Hydrogen cannot be exhausted heedlessly from the anode out of the hydrogen supply system and the magnitude of the generated current cannot be changed for the purpose of reducing the hydrogen pressure. Therefore, the hydrogen pressure cannot be reduced using the control input to the hydrogen pressure valve under a condition of decreasing pressure. The generated current and opening the purge valve are the only available ways of reducing the pressure.

Figure 8 shows the configuration of the differential pressure control system that was constructed to take this assumption into account and includes both the hydrogen pressure controller and the air pressure controller.

It will be noted that $\boldsymbol{G}$ in Fig. 8 is derived using a $\boldsymbol{B}$ matrix and assuming a constant value of $p_{r m}$ in Eq. (8). $r_{a f}$ is the reference air mass flow rate, and the larger of either the hydrogen pressure $y_{h p}$ or the reference pressure $r_{a p 0}$ is taken as the reference air pressure $r_{a p}$. When the reference air pressure $r_{a p}$ is set to $r_{a p 0}$, the air pressure response is predicted using Eq. (27). The predicted signal $y_{p}$ is input into the PID controller as the reference of the hydrogen pressure. With this system configuration, the hydrogen supply system follows the predicted air pressure $y_{p}$ relative to the reference pressure $r_{a p 0}$.

The notation i denotes the generated current, which consumes the hydrogen according to Eq. (2) and reduces the hydrogen pressure. All cases examined under the assumptions above are explained below. The block labeled MAX in Fig. 8 indicates the block that outputs the largest signal among the input signals. 


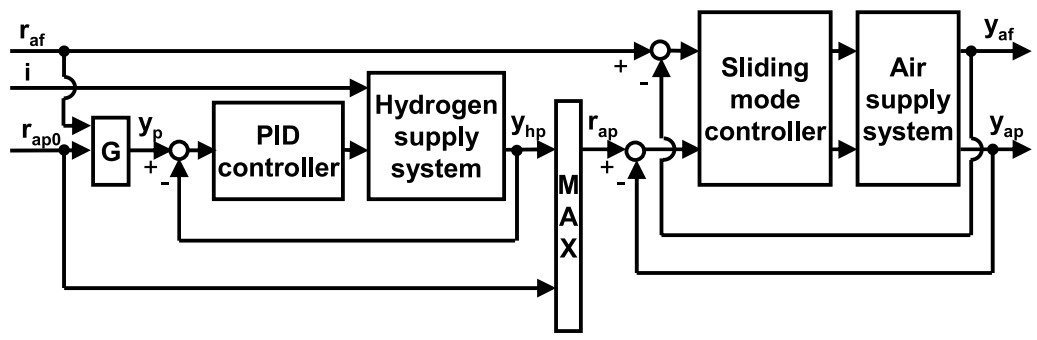

Fig. 8 Configuration of differential pressure control system

\subsection{The case of increasing pressure}

The highly responsive hydrogen pressure follows the predicted value of the air pressure that has low responsiveness. An examination of the reference air pressure $r_{a p}$ under an increasing pressure condition indicates that $r_{a p 0}>y_{p}$ is valid because the hydrogen pressure is following the predicted air pressure value $y_{p} ; r_{a p 0}$ is selected as the reference air pressure $r_{a p}$. The differential pressure control system configured for an increasing pressure condition is selected as Fig. 9.

The hydrogen supplied from the high pressure hydrogen tank, displays a much higher response. Therefore, the dynamics of the hydrogen pressure is ignored. As a result, the differential pressure becomes zero under an ideal state.

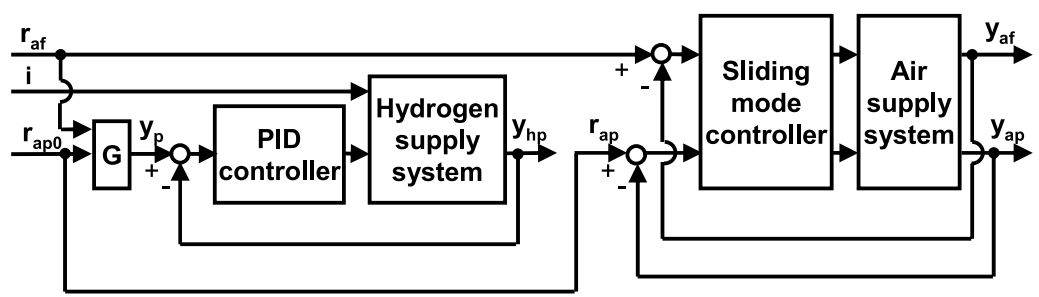

Fig. 9 Configuration of differential pressure control system for increasing pressure

\subsection{The case of air pressure displaying a higher response under a condition of decreasing} pressure

The tracking performance of the hydrogen pressure displays a lower response than the air pressure in this case, because the generated current decreases in a short period of time and does not sufficiently consume the supplied hydrogen. When the responsiveness of the hydrogen supply system is lower than that of the air supply system, the hydrogen pressure follows the predicted air pressure value $y_{p}$, which has too high a response for the hydrogen pressure to follow sufficiently. The hydrogen pressure $y_{h p}$ obtained as a result is made the reference air pressure $r_{a p}$, resulting in a configuration where the air pressure follows the hydrogen pressure $y_{h p}$ which is set to the reference value $r_{a p}$. The differential pressure is kept within the specified range. The configuration of the control system is shown in Fig. 10.

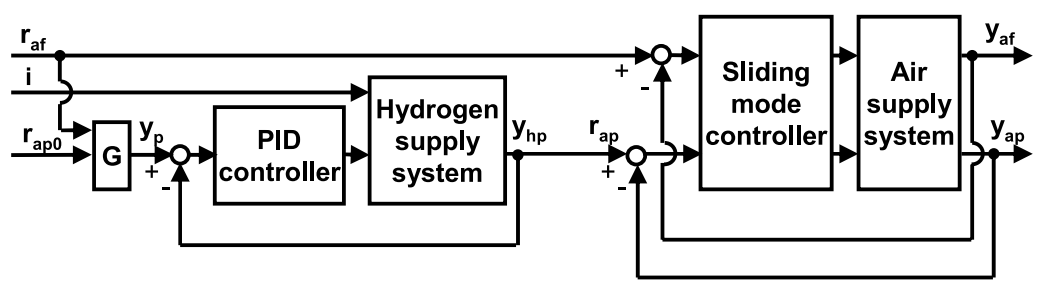

Fig. 10 Configuration of differential pressure control system for a condition of decreasing pressure 
5.3. The case of hydrogen pressure displaying a higher response under a condition of decreasing pressure

The tracking performance of the hydrogen pressure displays a higher response than the air pressure in this case, because the generated current decreases a little more slowly and consumes the hydrogen sufficiently. The hydrogen pressure control system follows the predicted air pressure value $y_{p}$, just as it does under a condition of increasing pressure. The hydrogen pressure $y_{h p}$ is adopted as the reference air pressure $r_{a p}$, because the reference pressure $r_{a p 0}$ is smaller than $y_{h p}$ and $y_{p}$ in this situation. $y_{p}$ is guided by a small reference pressure value $r_{a p 0}$ and $y_{h p}$ follows $y_{p}$ with a delay time. Therefore, in this situation the relationship of $r_{a p 0}<y_{p}<y_{h p}$ is satisfied. Strictly speaking, the predicted value $y_{p}$ and the air pressure $y_{a p}$ no longer coincide. However, because the hydrogen pressure $y_{h p}$ is controlled to follow the predicted air pressure value $y_{p}$, it does not respond in such a way that the differential pressure increases more than the air pressure. Therefore, the control system can keep the differential pressure within the specified range. The configuration of the control system is shown in Fig. 9.

\section{Experimental results}

The validity of the control system that was designed as described in the preceding sections was verified experimentally using Fig. 1, however an electric instrument was used instead of the motor. The electric instrument extracted current from the fuel cell according to the reference given for current generation. The reference was given manually based on a program, and the pressure references were determined according to the current generation reference. The pressure references were increased as the generated current level increased.

Figure 11 shows the experimental results obtained for an increasing pressure condition. The results indicate that the range of variation in the differential hydrogen and air pressures was kept to within 5\% of the reference values. Figure 11 represents a situation where a driver depresses the accelerator pedal quickly. The fuel cell system must increase the generated current from a few amperes to about 300A in approximately a second. Although hydrogen and air should be fed to the fuel cell as fast as possible, air is compressed using the compressor and hydrogen is supplied from the high pressure hydrogen tank. Generally, there is apt to be more delay in the air pressure response to the references than in the hydrogen pressure response. The proposed method makes the hydrogen pressure follow the predicted air pressure, taking into account the time delay of the air pressure.

The experimental results for decreasing pressure conditions are shown in Fig. 12 and Fig. 13. Figure 12 presents the results for the case in section 5.2, and Fig. 13 shows the results for the situation in section 5.3. In these cases, the control system was not configured so that the predicted air pressure and the air response coincided strictly. Consequently, the differential pressure values were larger compared with those of the increasing pressure condition. Yet, the range of variation was kept to within $15 \%$ of the reference values. These experiments were implemented from the maximum current to a level of a few amperes. The generated current in Fig. 12 decreases in a short period of time. Therefore, the consumption of hydrogen by current generation did not progress much and the hydrogen pressure was reduced slowly by the current of a few amperes. In contrast, current generation in Fig. 13 encouraged the consumption of hydrogen, which is why the hydrogen pressure displayed a higher response in this case. 

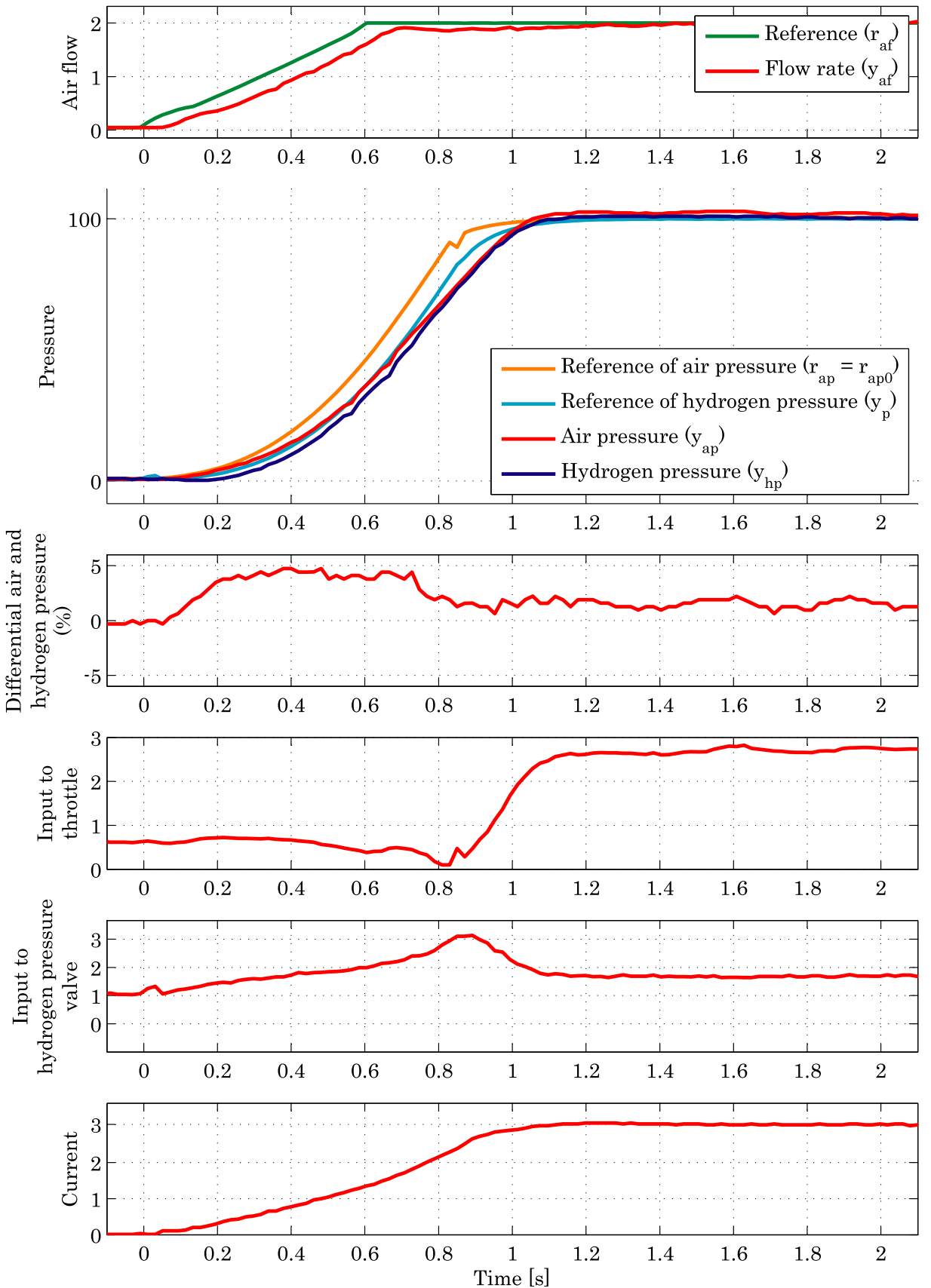

Fig. 11 Experimental results for increasing pressure condition 

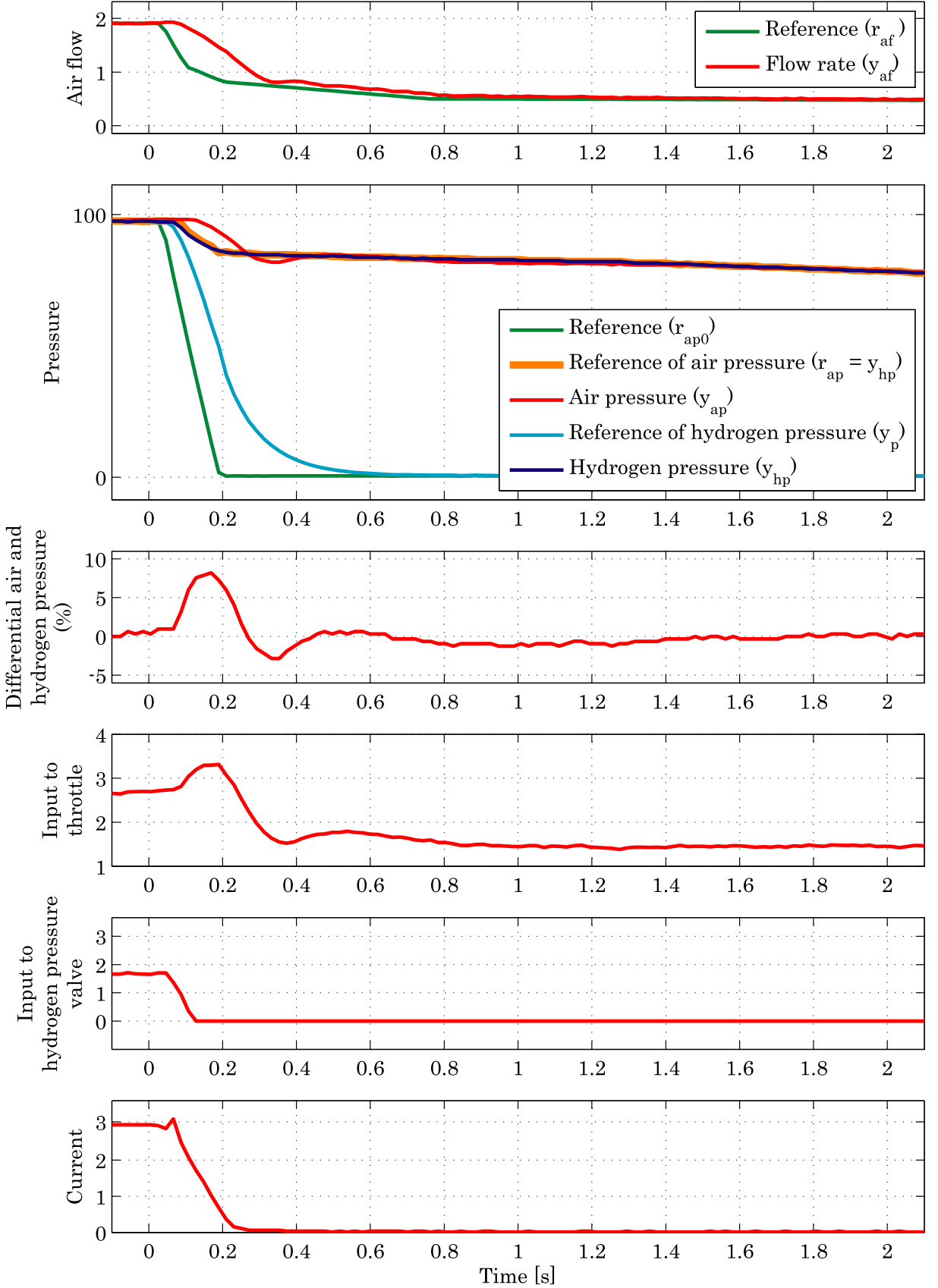

Fig. 12 Experimental results for decreasing pressure condition 

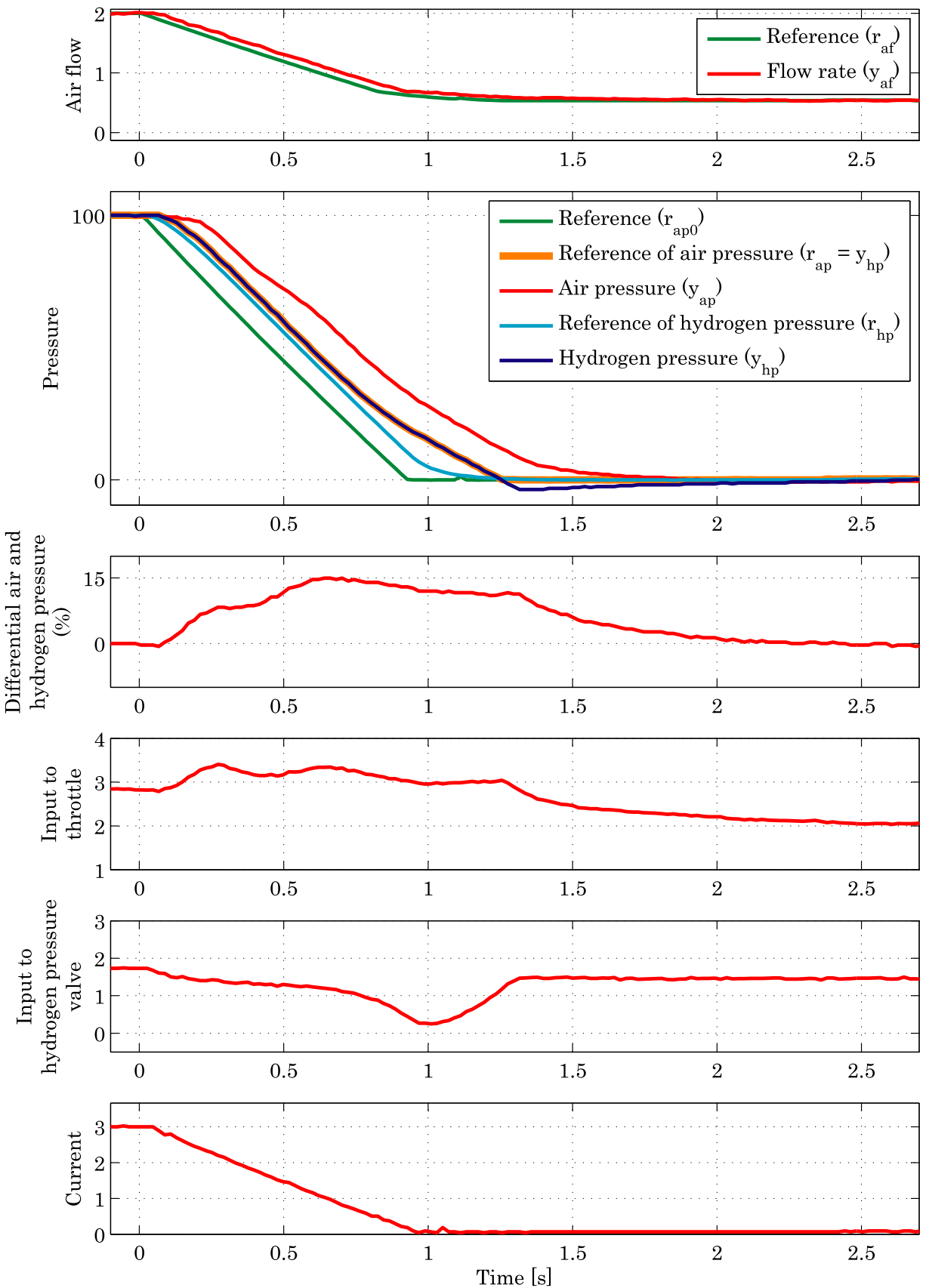

Fig. 13 Experimental results for decreasing pressure condition

\section{Conclusions}

This paper has proposed a method for controlling differential hydrogen and air pressures in a fuel cell system in which the responsiveness of the hydrogen pressure and air pressure varies depending on the operating conditions. The validity of the proposed control method was verified experimentally.

In future work, the effect on pressure of the consumption of hydrogen and oxygen during power generation will be incorporated in the control system as a known disturbance and efforts will be made to improve control performance further. 


\section{References}

( 1 ) Working Groups of the IPCC, Climate Change 2007 -Synthesis Report, (2007).

( 2 ) Asai, Y. and Takahashi, N., Two variables and Two-Degree-of-Freedom Sliding Mode Control for Pneumatic Pressure and Flow of Fuel Cell System, Transactions of the Japan Society of Mechanical Engineers, Series C, Vol. 75, No. 756, (2009), pp. 2311-2318

( 3 ) Jay, T. P., Anna, G. S. and Huei, P., Control of Fuel Cell Power Systems, (2004), pp.2126, Springer.

( 4 ) Chan-Chiao, L., Min-Joong, K., Huei, P., and Jessy, W. G., System-Level Model and Stochastic Optimal Control for a PEM Fuel Cell Hybrid Vehicle, ASME Journal of Dynamic Systems, Measurement and Control, Vol.128, No. 4, (2006), pp.878-890

( 5 ) Johannes, W. R., David, C. R. and Utz-Jens, B., Control of a Fuel Cell Air Supply Module (ASM), SAE Technical Paper Series 2004-01-1009, (2004).

( 6 ) Michael, A. D., Jorg, W, Harald, A. and Eberhard, P. H., Model-based control of cathode pressure and oxygen excess ratio of a PEM fuel cell system, Journal of Power Sources, Vol.176, No.2, (2008), pp. 515-522

( 7 ) Fu-Cheng, W., Yee-Pien, Y., Chi-Wei, H., Hsin-Ping, C. and Hsuan-Tsung, C., System identification and robust control of a portable proton exchange membrane fuel-cell system, Journal of Power Sources, Vol.164, No.2, (2007), pp. 704-712

( 8 ) Nonami, K., Nishimura, H., Hirata, M., Control System Design by MATLAB, (1998), Tokyo Denki University Press(in Japanese)

( 9 ) Nonami, K., H. Tian, Sliding Mode Control, Corona Corporation(in Japanese), (1994).

(10) Maeda, H. and Sugie, T., System Control Theory for Advanced Control, (1990), Asakura Shoten(in Japanese) 